\title{
Study of thermal monopoles in lattice QCD
}

\author{
Vitaly Bornyakov* \\ IHEP, Protvino \\ E-mail: vitaly.bornyakov@ihep.ru
}

\section{A.G. Kononenko}

Moscow State University, Physics Department, Moscow, Russia

E-mail: agkono@gmail.com

\section{V.K. Mitrjushkin}

Joint Institute for Nuclear Research, 141980, Dubna, Russia and Institute of Theoretical and Experimental Physics, 117259 Moscow, Russia

E-mail: vmitratheor.jinr.ru

The properties of the thermal Abelian color-magnetic monopoles in the maximally Abelian gauge are studied in the vicinity of the confinement-deconfinement phase transition in the lattice $S U(3)$ gluodynamics and lattice QCD. We compute the density and interaction parameters of the thermal monopoles. We find that the density of the thermal monopoles $\rho(T)$ jumps up near the transition temperature $T_{c}$. Additionally we present new results on the percolation transition in $S U(3)$ gluodynamics which is known to coincide in gluodynamics with the confinement-deconfinement phase transition.

31st International Symposium on Lattice Field Theory LATTICE 2013

July 29 âĂS August 3, 2013

Mainz, Germany

\footnotetext{
* Speaker.
} 


\section{Introduction}

The nonperturbative properties of the nonabelian gauge theories, e.g., confinement, confinementdeconfinement transition, chiral symmetry breaking, etc. are closely related to the Abelian monopoles defined in the maximally Abelian gauge $(M A G)[1,2]$.

It has been recently argued that the MAG is a proper Abelian gauge to find gauge invariant monopoles since t'Hooft-Polyakov monopoles can be identified in this gauge by the Abelian flux, but this is not possible in other Abelian gauges [3].

In recent papers $[4,5]$ it has been suggested that color-magnetic monopoles contribution can explain the strong interactions in the quark-gluon matter which were found in heavy ion collisions experiments [6]. These proposals inspired studies of the properties and possible roles of the monopoles in the quark-gluon phase $[7,8,9,10,11,12,13,14,15,16]$.

In Ref. [5] it has been shown that thermal monopoles in Minkowski space are associated with Euclidean monopole trajectories wrapped around the temperature direction of the Euclidean volume. So the density of the monopoles in the Minkowski space is given by the average of the absolute value of the monopole wrapping number. First numerical investigations of the wrapping monopole trajectories were performed in $S U(2)$ Yang-Mills theory at high temperatures in Refs. [17] and [18]. A more systematic study of the thermal monopoles was performed in Ref. [9]. It was found in [9] that the density of monopoles is independent of the lattice spacing, as it should be for a physical quantity. The density-density spatial correlation functions were also computed in [9]. It was shown that there is a repulsive (attractive) interaction for a monopole-monopole (monopoleantimonopole) pairs, which at large distances might be described by a screened Coulomb potential with a screening length of the order of $0.1 \mathrm{fm}$. In Ref. [10] it was proposed to associate the respective coupling constant with a magnetic coupling $\alpha_{m}$. In the paper [12] trajectories which wrap more than one time around the time direction were investigated. It was shown that these trajectories contribute significantly to a total monopole density at $T$ slightly above $T_{c}$. It was also demonstrated that Bose condensation of thermal monopoles, indicated by vanishing of the monopole chemical potential, happens at temperature very close to $T_{c}$.

The quantitatively precise determination of such parameters as monopole density, monopole coupling and others is necessary, in particular, to verify the conjecture [4] that the magnetic monopoles are weakly interacting (in comparison with electrically charged fluctuations) just above transition but become strongly interacting at high temperatures.

So far lattice studies of the thermal monopoles were mostly made for $S U(2)$ gluodynamics. First results for $S U$ (3) gluodynamics and QCD were presented in our previous paper [19] In this paper we present new results of our study of the thermal monopoles in the $S U(3)$ gluodynamics concentrating on the vicinity of the confinement-deconfinement phase transition. To avoid systematic effects due to Gribov copies we use the gauge fixing procedure as in Ref. [20] with 10 gauge copies.

In paper [21] the thermal monopoles were investigated with new definition of MAG. The results obtained were in qualitative agreement with results of Ref. [19]. 


\section{Definitions and simulation details}

MAG is determined by the gauge condition [1]

$$
\sum_{c \neq 3,8}\left(\partial_{\mu} \delta_{a c}+\sum_{b=3,8} f_{a b c} A_{\mu}^{b}(x)\right) A_{\mu}^{c}(x)=0, \quad a \neq 3,8
$$

Solutions of this equation are extrema (over $g$ ) of the functional $F_{\mathrm{MAG}}\left[A^{g}\right]$

$$
F_{\mathrm{MAG}}[A]=\frac{1}{V} \int d^{4} x \sum_{a \neq 3,8}\left[A_{\mu}^{a}(x)\right]^{2}
$$

Abelian projection means discarding offdiagonal components from the observables

$$
\sum_{a} A_{\mu}^{a}(x) T^{a} \rightarrow A_{\mu}^{3}(x) T^{3}+A_{\mu}^{8}(x) T^{8}
$$

On a lattice MAG gauge fixing functional and Abelian projection are of the form [2]

$$
F(U)=\frac{1}{V} \sum_{x, \mu, i}\left(\left|U_{\mu}(x)^{i i}\right|^{2}\right), \quad U_{\mu}(x) \rightarrow u_{\mu}(x) \in U(1)^{2}
$$

After Abelian projection one can define magnetic currents:

$$
j_{\mu}^{i} \equiv \frac{1}{4 \pi} \varepsilon_{\mu v \rho \sigma} \partial_{\nu} \bar{\Theta}_{\rho \sigma}^{(i)}=-\frac{1}{2} \varepsilon_{\mu v \rho \sigma} \partial_{\nu} m_{\rho \sigma}^{(i)}, i=1,2,3
$$

were $\bar{\Theta}_{\rho \sigma}^{(i)}$ is lattice Abelian field strength. The magnetic currents satisfy the constraint

$$
\sum_{a} j_{\mu}^{i}(x)=0
$$

on any link $\{x, \mu\}$ of the dual lattice. Furthermore magnetic currents are conserved and form closed loops.

Thermal monopoles are defined as clusters of magnetic currents wrapped in the temperature dimension. Wrapping number for given cluster $N_{w r}^{i}$ is equal to:

$$
N_{w r}^{i}=\frac{1}{3 L_{t}} \sum_{j_{4}^{i}(x) \in \text { cluster }} j_{4}^{i}(x)
$$

Then respective density is

$$
\rho=\frac{\left\langle\sum_{\text {clusters }, a}\left|N_{w r}^{i}\right|\right\rangle}{3 L_{s}^{3} a^{3}}
$$

One can also define the densities $\rho_{k}$ of the thermal monopoles wrapped $k$ times.

$S U$ (3) lattice gluodynamics was simulated with Wilson action. Configurations of $N_{f}=2$ lattice QCD were produced on lattices $32^{3} \cdot 12$ with non-perturbatively $O(a)$ improved Wilson fermionic action at $\beta=5.25$. It had been found by DIK collaboration [22] that at crossover $T_{c} \approx$ $200 \mathrm{MeV}$ and $m_{\pi} \approx 400 \mathrm{MeV}$. 


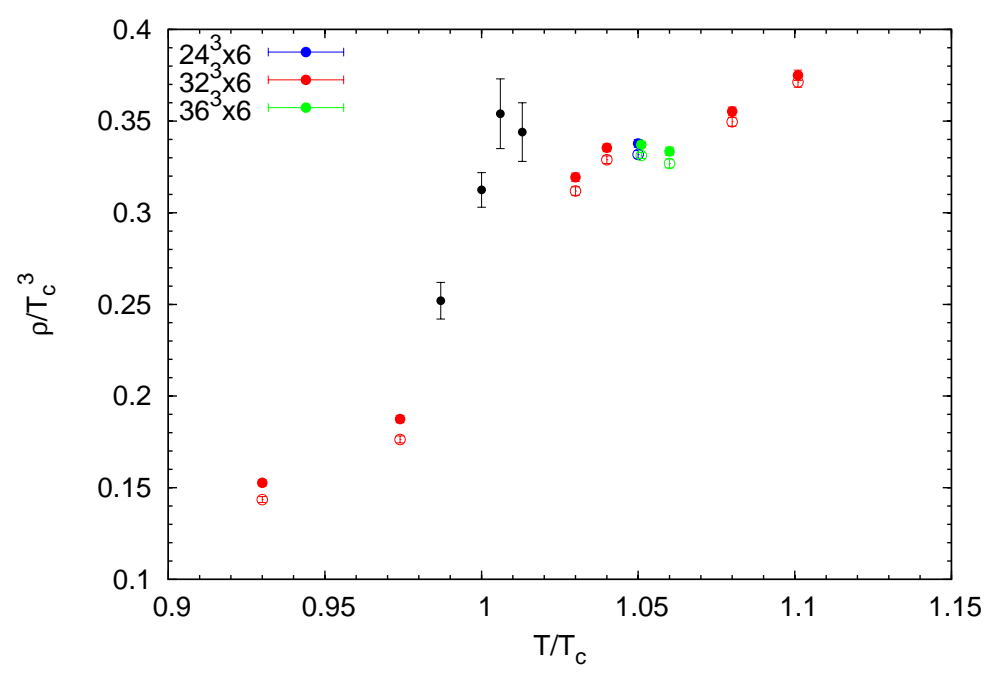

Figure 1: Full monopole density $\rho(T)$ (full symbols) and density $\rho_{1}(T)$ (empty symbols) in SU(3) gluodynamics vs $T / T_{c}$. Full monopole density for full QCD (black symbols) is shown for comparison.

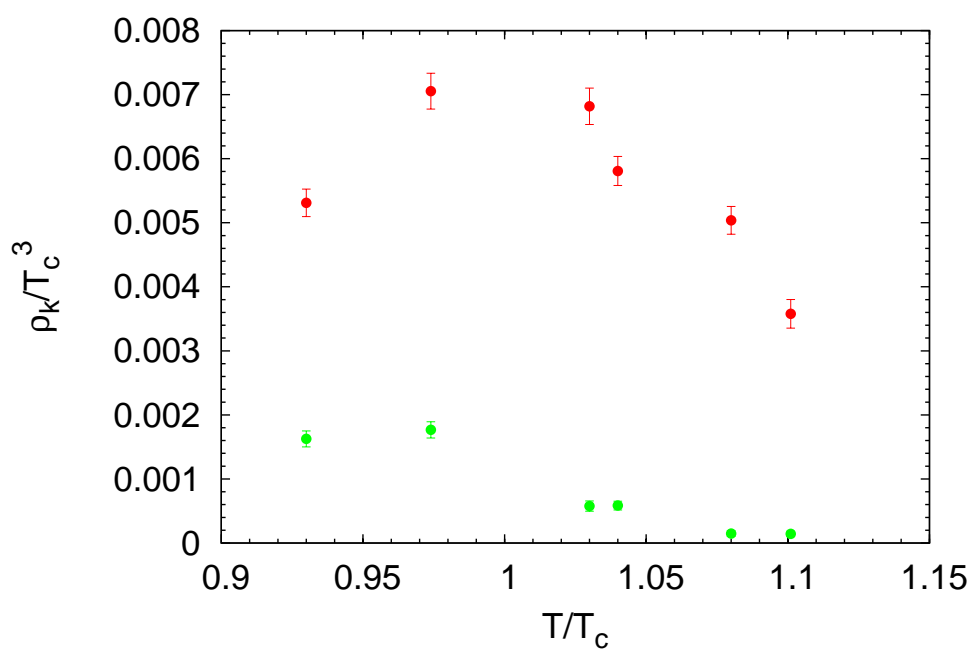

Figure 2: Monopole density $\rho_{k}(T)$ for $k=2$ (red) and $k=3$ (green) in $S U(3)$ gluodynamics vs $T / T_{c}$.

\section{Results}

In Figure 1 we show density $\rho(T)$ defined in eq. 2.8 and density $\rho_{1}(T)$ for thermal monopoles wrapped one time as function of the ratio $T / T_{c}$ for temperatures below and above $T_{c}$. The data indicate volume independence of both densities. It can also be seen that $\rho_{1}(T)$ is a main contribution to $\rho(T)$ at all temperatures. The important new observation is that both densities jump up at the transition point. Thus density $\rho_{1}(T)$ is indicates the phase transition. One can imagine that this sharp increase of $\rho_{1}(T)$ is due to evaporation of the monopole condensate, existing in the confinement phase, or, in other words, many clusters with $N_{w r}=1$ appear from disintegrated percolating 
cluster. In Figure 1 we show for comparison the density $\rho(T)$ computed for full QCD. One can see that in this case the density also grows fast near the phase transition.

The behavior of the densities $\rho_{k}(T)$ for $k=2$ and 3, shown in Figure 2, is quite different. They increase below $T_{c}$ and decrease above $T_{c}$.

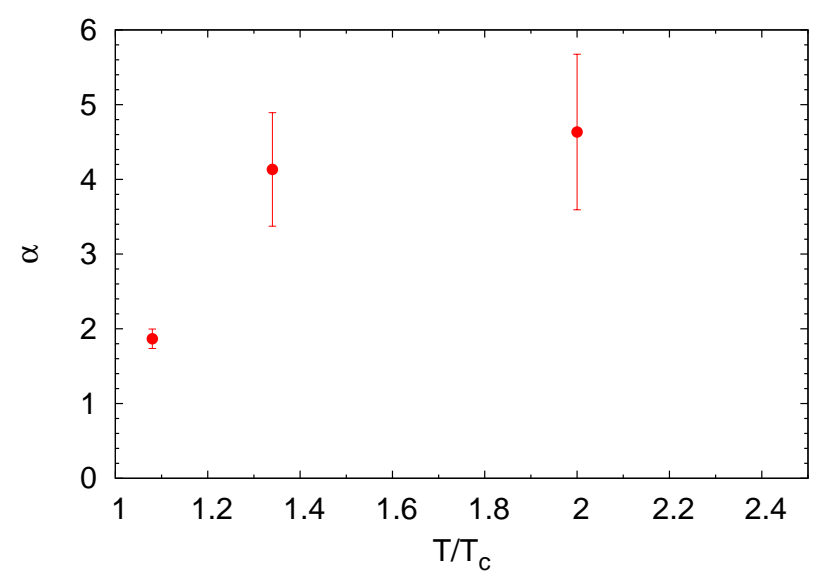

Figure 3: Magnetic coupling $\alpha_{M}$ in $\mathrm{SU}(3)$ gluodynamics vs $T / T_{c}$.

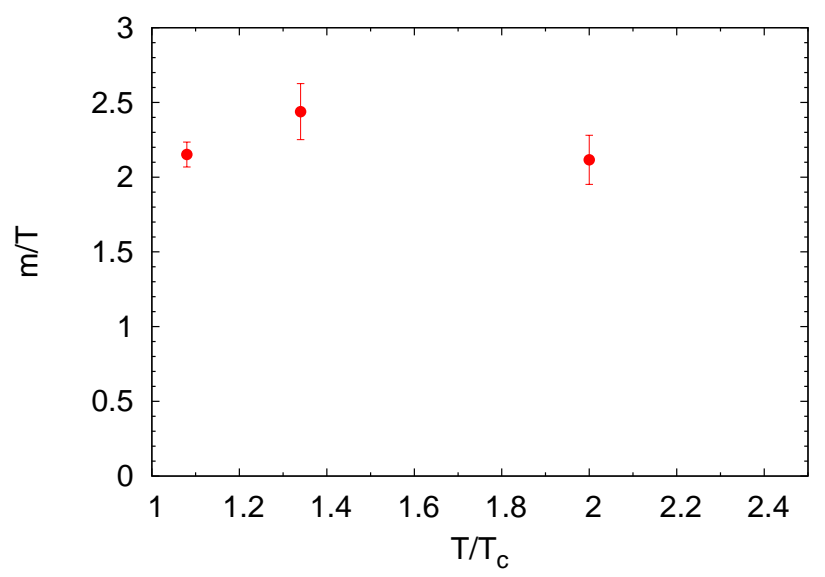

Figure 4: Screening mass $m_{D}$ in SU(3) gluodynamics vs $T / T_{c}$.

We also computed the correlators for charges of same sign $\left(g_{M M}(r)\right)$ and for charges of opposite sign $\left(g_{A M}(r)\right)$ :

$$
\begin{aligned}
& g_{M M}(r)=\frac{\left\langle\rho_{M}^{a}(0) \rho_{M}^{a}(r)\right\rangle}{2 \rho_{M}^{b} \rho_{M}^{b}}+\frac{\left\langle\rho_{A}^{a}(0) \rho_{A}^{a}(r)\right\rangle}{2 \rho_{A}^{b} \rho_{A}^{b}} \\
& g_{A M}(r)=\frac{\left\langle\rho_{A}^{a}(0) \rho_{M}^{a}(r)\right\rangle}{2 \rho_{A}^{b} \rho_{M}^{b}}+\frac{\left\langle\rho_{M}^{a}(0) \rho_{A}^{a}(r)\right\rangle}{2 \rho_{A}^{b} \rho_{M}^{b}}
\end{aligned}
$$

The correlators were fitted to functions $[9,10]$

$$
g_{M M, A M}(r)=e^{-U(r) / T},
$$


where

$$
U(r)=\frac{\alpha_{m}}{r} e^{-m_{D} r}
$$

In Figure 3 and Figure 4 we show dependence of the parameters $\alpha_{m}$ and $m_{D}$ on temperature. It can be seen that $\alpha_{m}$ increases fast above $T_{c}$ and then flattens. $m_{D} / T$ increases slightly near $T_{c}$ and starts to decrease at higher temperatures. These results are in agreement with results obtained for $S U(2)$ gluodynamics $[10,15]$.

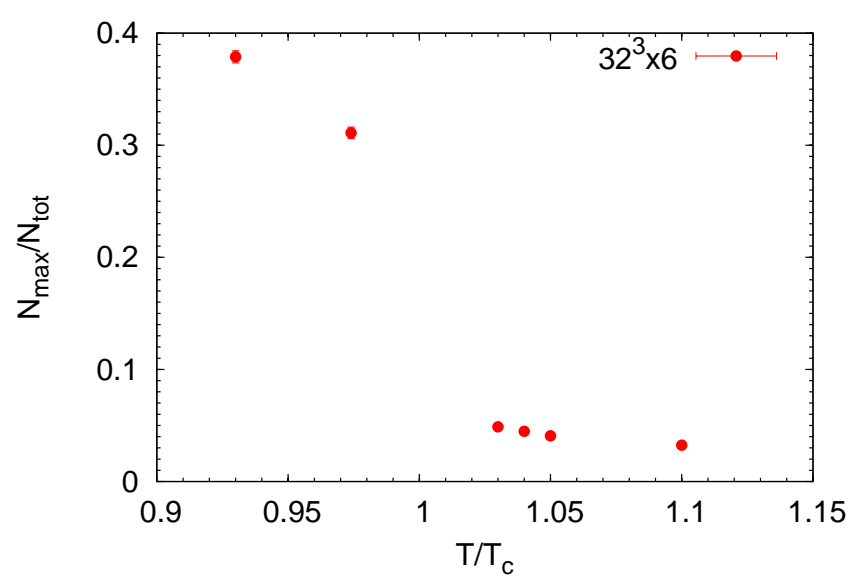

Figure 5: Percolation transition order parameter.

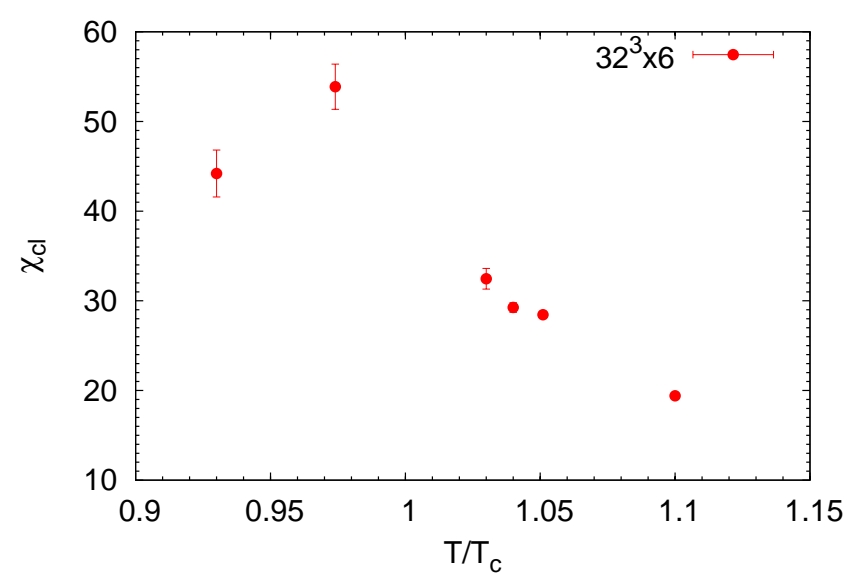

Figure 6: Nonpercolating monopole cluster average size - 'susceptibility' $\chi_{c l}$

Additionally we have studied the percolation of the magnetic currents at $T_{c}$ in $S U(3)$ gluodynamics. In Figure 5 the ration of the average size of the maximal cluster to the full number of the magnetic currents, i.e. order parameter of the percolation transition is depicted. In Figure 6 the nonpercolating cluster average size which is often called susceptibility is shown. Both observables indicate that the percolation transition coincides with the phase transition. 


\section{Conclusions}

We have found that density of thermal monopoles both in $S U(3)$ gluodynamics and in QCD grows fast in the vicinity of the transition (crossover) point. This is determined by the $\rho_{1}(T)$ contribution alone. Our data indicate volume independence of the densities $\rho(T)$ and $\rho_{1}(T)$. The magnetic coupling $\alpha_{m}$ and screening mass $m_{D}$ show qualitatively same behavior as in $S U(2)$ gluodynamics.

\section{Acknowledgements}

This work has been supported by grant RFBR 13-02-01387-a.

\section{References}

[1] G. 't Hooft, Nucl. Phys. B 190 (1981) 455.

[2] A. S. Kronfeld, M. L. Laursen, G. Schierholz and U. J. Wiese, Phys. Lett. B 198 (1987) 516.

[3] C. Bonati, A. Di Giacomo, L. Lepori and F. Pucci, Phys. Rev. D 81 (2010) 085022 [arXiv:1002.3874 [hep-lat]].

[4] J. Liao and E. Shuryak, Phys. Rev. C 75 (2007) 054907 [hep-ph/0611131].

[5] M. N. Chernodub and V. I. Zakharov, Phys. Rev. Lett. 98 (2007) 082002 [hep-ph/0611228].

[6] J. Adams et al. [STAR Collaboration], Nucl. Phys. A 757 (2005) 102 [nucl-ex/0501009].

[7] E. Shuryak, Prog. Part. Nucl. Phys. 62 (2009) 48 [arXiv:0807.3033 [hep-ph]].

[8] C. Ratti and E. Shuryak, Phys. Rev. D 80 (2009) 034004 [arXiv:0811.4174 [hep-ph]].

[9] A. D’Alessandro and M. D’Elia, Nucl. Phys. B 799 (2008) 241 [arXiv:0711.1266 [hep-lat]].

[10] J. Liao and E. Shuryak, Phys. Rev. Lett. 101 (2008) 162302 [arXiv:0804.0255 [hep-ph]].

[11] M. N. Chernodub, A. D’Alessandro, M. D’Elia and V. I. Zakharov, arXiv:0909.5441 [hep-ph].

[12] A. D’Alessandro, M. D’Elia and E. V. Shuryak, Phys. Rev. D 81 (2010) 094501 [arXiv:1002.4161 [hep-lat]].

[13] V. G. Bornyakov and V. V. Braguta, Phys. Rev. D 84 (2011) 074502 [arXiv:1104.1063 [hep-lat]].

[14] V. G. Bornyakov and V. V. Braguta, Phys. Rev. D 85 (2012) 014502 [arXiv:1110.6308 [hep-lat]].

[15] V. G. Bornyakov and A. G. Kononenko, Phys. Rev. D 86 (2012) 074508 [arXiv:1111.0169 [hep-lat]].

[16] V. V. Braguta and A. Y. .Kotov, Phys. Rev. D 86 (2012) 014511 [arXiv:1208.5344 [hep-lat]].

[17] V. G. Bornyakov, V. K. Mitrjushkin and M. Muller-Preussker, Phys. Lett. B 284 (1992) 99.

[18] S. Ejiri, Phys. Lett. B 376 (1996) 163 [hep-lat/9510027].

[19] V. G. Bornyakov, A. G. Kononenko and V. K. Mitrjushkin, PoS ConfinementX (2012) 048

[20] V. G. Bornyakov et al. [DIK Collaboration], Phys. Rev. D 70 (2004) 074511 [hep-lat/0310011].

[21] C. Bonati, M. D'Elia, [arXiv:1308.0302 [hep-lat]].

[22] V. G. Bornyakov, R. Horsley, S. M. Morozov, Y. Nakamura, M. I. Polikarpov, P. E. L. Rakow, G. Schierholz and T. Suzuki, Phys. Rev. D 82 (2010) 014504 [arXiv:0910.2392 [hep-lat]]. 